Research Article

\title{
Study on the Composite Structure of Aluminum Foam-Filled Thin-Walled Metal Tube to Reduce the Charge Overload inside the Projectile during the Penetration Process
}

\author{
Fudi Liang $(\mathbb{D}$, Zengyou Liang $(\mathbb{D}$, and Dezhi Deng $\mathbb{1}$ \\ College of Mechatronic Engineering, North University of China, Taiyuan, China \\ Correspondence should be addressed to Zengyou Liang; liangzy@nuc.edu.cn
}

Received 20 June 2020; Revised 14 September 2020; Accepted 17 September 2020; Published 12 October 2020

Academic Editor: Jiang Jin

Copyright ( 2020 Fudi Liang et al. This is an open access article distributed under the Creative Commons Attribution License, which permits unrestricted use, distribution, and reproduction in any medium, provided the original work is properly cited.

\begin{abstract}
When a projectile penetrates a target at high speed, the charge loaded inside the projectile usually bears a high overload, which will consequently severely affect its performance. In order to reduce the overload of the charge during the penetration process, the structure of the projectile was improved by adding two buffers at both ends of the charge. In this study, the mathematical expressions were first gained about the axial buffering force generated by the thin-walled metal tube, aluminum foam, and the composite structure of aluminum foam-filled thin-walled metal tube when they were impacted by the high-speed mass block through reasonable assumptions and stress analysis. During the experiment on the high-speed projectile penetrating reinforced concrete target, the acceleration curve of the charge and the projectile body were obtained. The results show that the maximum overload that the charge was subjected to during the launch and penetration process was significantly reduced, and the change in overload, which the charge was subjected to during the penetration process, was also less obvious.
\end{abstract}

\section{Introduction}

Earth penetrating weapon (EPW) is used to strike deep underground targets. The process of destroying the target can be divided into two steps: first of all, EPW breaks through the protective layer of the target, when the EPW must have a high speed to make sure that it can get into the target; then, the EPW detonates the charge with the action of fuse to destroy the target.

The charge suffers a very high shock overload when the EPW penetrates the protective layer at a high speed. There are studies showing that the charge column deformed with cracks while suffering the high overload, which seriously affected its performance. Muthig and Arnold [1] shot the concrete target vertically with the cannon, recovered the ks22a charge inside it, observed its morphological change, and tested its performance. The results show that the charge was broken and the sensitivity of the explosive was obviously reduced.

The thin-walled metal tube and aluminum foam could absorb energy of the impact and collision through the elastic-plastic deformation and have the advantages of having the simple structure and stable operation [2-4]. The composite structure of aluminum foam-filled metal thinwalled tube can not only make up for the defect of instability and collapse of aluminum foam when used alone but also solve the problem of poor nonaxial load-bearing capacity of metal thin-walled tube under the impact. This composite structure has huge application potential in aerospace, rail transit, and other fields $[5,6]$ for its independent carrying capacity, strong stability, high energy absorption efficiency, and working in extreme environments.

The main content of this research is how to apply the advantages of foam aluminum and thin-walled metal tubes in the field of energy absorption to EPW to reduce the impact load of the charge during the penetration process. The methodology applied in this research is theoretical analysis and experimental verification: first of all, a theoretical analysis was conducted on the stress conditions of thin-walled metal tube, aluminum foam, and composite structure when they were impacted by high-speed mass 
block and expressions of the axial buffering force were presented in Section 2; then, we designed the thin-walled metal tube buffer and the composite-structured buffer, which were added to the ends of the charge. Finally, an experiment was conducted on the EPW penetration into reinforced concrete to verify the effectiveness of buffers for reducing the overload of the charge.

\section{Theoretical Analysis}

2.1. Buffering Force of Thin-Walled Metal Tube under HighSpeed Axial Impact. Subject in this section is shown in Figure 1. The high-speed impact mentioned in this section refers to the impact of the mass block speed ranging from $400 \mathrm{~m} / \mathrm{s}$ to $800 \mathrm{~m} / \mathrm{s}$. The formula of tube buffering force is deduced by using the principal stress method. Steel was selected as the material of the metal tube; von Mises yield condition as the yield condition of the metal tube and Cowper-Symonds constitutive model as the constitutive model of the metal tube.

In order to obtain the formula of the buffering force, we made the following assumptions: the deformation of the mass block is ignored;

(1) The stress of the metal tube along the direction of wall thickness is evenly distributed when the mass block hits the metal tube

(2) Ignore the elastic deformation of the metal tube

(3) The friction coefficient between the metal tube and the mass block does not change in the whole process
The stress condition of the tube is shown in Figure 2(a) and the stress state at arbitrary radius $(r)$ is shown in Figure 2(b).

The force balance equation perpendicular to the tube wall:

$$
\begin{gathered}
\sigma_{r} \cdot r \cdot d \theta \cdot d l-2 \sigma_{\theta} \cdot \sin \frac{d \theta}{2} \cdot t \cdot d l \cdot \cos \alpha \\
-\rho \cdot r \cdot t \cdot d \theta \cdot d l \cdot a=0 .
\end{gathered}
$$

The kinematic balance equation:

$$
\frac{1}{2} a T^{2}=R_{2}-R_{1} \text {. }
$$

Time:

$$
T=\frac{l \cdot \cos \alpha}{v}
$$

Get the acceleration:

$$
a=\frac{2 v^{2} \cdot\left(R_{2}-R_{1}\right)}{l^{2} \cdot \cos ^{2} \alpha} .
$$

Substituting equation (4) into equation (1), we obtained equation (5) as follows:

$$
\sigma_{r}=\frac{\sigma_{\theta} \cdot t \cdot \cos \alpha}{r}+\frac{2 \cdot \rho \cdot t \cdot v^{2}\left(R_{2}-R_{1}\right)}{l^{2} \cdot \cos ^{2} \alpha}
$$

The force balance equation parallel to the tube wall:

$$
\left(\sigma_{L}+d \sigma_{L}\right) \cdot(r+d r) \cdot(t+d t) \cdot d \theta-\sigma_{L} \cdot r \cdot t \cdot d \theta+2 \sigma_{\theta} \cdot \sin \frac{d \theta}{2} \cdot t \cdot d l \cdot \sin \alpha+f \cdot \sigma_{r} \cdot r \cdot d \theta \cdot d l=0
$$

Substituting $d l=d r / \sin \alpha$ into equation (6), ignore the infinitely small:

$$
\frac{d\left(\sigma_{L} \cdot r \cdot t\right)}{d r}+\sigma_{\theta} \cdot t+\frac{f \cdot \sigma_{r} \cdot r}{\sin \alpha}=0 .
$$

Ignore the change of thickness, $d t / d r=0$ :

$$
\frac{d \sigma_{L} \cdot r \cdot t}{d r}+\sigma_{L} \cdot t+\sigma_{\theta} \cdot t+\frac{f \cdot \sigma_{r} \cdot r}{\sin \alpha}=0 .
$$

Substituting equation (5) into equation (8),

$$
\begin{gathered}
\frac{d \sigma_{L} \cdot r}{d r}+\sigma_{L}+\sigma_{\theta}+f \cdot \sigma_{\theta} \cdot \operatorname{ctg} \alpha+\frac{2 f \cdot r \cdot \rho \cdot v^{2} \cdot\left(R_{2}-R_{1}\right)}{l^{2} \cdot \cos ^{2} \alpha \cdot \sin ^{2} \alpha}=0, \\
\sigma_{1}=\sigma_{\theta}, \\
\sigma_{2}=\sigma_{r}, \\
\sigma_{3}=-\sigma_{L} .
\end{gathered}
$$

Mises yield criterion:

$$
\sigma_{1}-\sigma_{3}=1.15 \sigma_{d}
$$

According to Cowper-Symonds' description of the yield strength of materials $[7,8]$ under dynamic loading, the relationship between the yield strength and strain rate of the material can be expressed by

$$
\sigma_{d}=\sigma_{s}\left[1+\left(\frac{\dot{\varepsilon}}{D}\right)^{1 / q}\right] .
$$

Combining equations (10)-(12),

$$
\sigma_{\theta}=1.15 \sigma_{s}\left[1+\left(\frac{\dot{\varepsilon}}{D}\right)^{1 / q}\right]-\sigma_{L} .
$$

Substituting equation (13) into equation (9), 


$$
\begin{gathered}
\frac{r \cdot d \sigma_{L}}{d r}+\sigma_{L}+(1+f \cdot \operatorname{ctg} \alpha)\left[1.15 \sigma_{s}+1.15 \sigma_{s} \cdot\left(\frac{\dot{\varepsilon}}{D}\right)^{1 / q}-\sigma_{L}\right]+\frac{2 f \cdot r \cdot \rho \cdot v^{2} \cdot\left(R_{2}-R_{1}\right)}{l^{2} \cdot \cos ^{2} \alpha \cdot \sin ^{2} \alpha}=0, \\
\frac{d \sigma_{L}}{\sigma_{L} \cdot f \cdot \operatorname{ctg} \alpha-1.15 \sigma_{s}(1+f \cdot \operatorname{ctg} \alpha)\left[1+(\dot{\varepsilon} / D)^{1 / q}\right]-\left(2 f \cdot r \cdot \rho \cdot v^{2} \cdot\left(R_{2}-R_{1}\right) / l^{2} \cdot \cos ^{2} \alpha \cdot \sin ^{2} \alpha\right)}=\frac{d r}{r} .
\end{gathered}
$$

Integrating both sides of equation (15), we obtain

$$
\frac{1}{f \cdot \operatorname{ctg} \alpha} \ln \left\{\sigma_{L} \cdot f \cdot \operatorname{ctg} \alpha-1.15 \sigma_{s}(1+f \cdot \operatorname{ctg} \alpha)\left[1+\left(\frac{\dot{\varepsilon}}{D}\right)^{1 / q}\right]-\frac{2 f \cdot r \cdot \rho \cdot v^{2} \cdot\left(R_{2}-R_{1}\right)}{l^{2} \cdot \cos ^{2} \alpha \cdot \sin ^{2} \alpha}\right\}=\ln r+\ln A .
$$

Boundary condition: Substituting equation (17) into equation (16),

$$
\begin{aligned}
& r=R_{2}, \\
& \sigma_{L}=0 .
\end{aligned}
$$

$$
\begin{gathered}
A=\frac{\left\{-1.15 \sigma_{s}(1+f \cdot \operatorname{ctg} \alpha)\left[1+(\dot{\varepsilon} / D)^{1 / q}\right]-2 f \cdot R_{2} \cdot \rho \cdot v^{2} \cdot\left(R_{2}-R_{1}\right) / l^{2} \cdot \cos ^{2} \alpha \cdot \sin ^{2} \alpha\right\}^{1 / f \cdot \operatorname{ctg} \alpha}}{R_{2}}, \\
B=-1.15 \sigma_{s}(1+f \cdot \operatorname{ctg} \alpha)\left[1+\left(\frac{\dot{\varepsilon}}{D}\right)^{1 / q}\right]-\frac{2 f \cdot R_{2} \cdot \rho \cdot v^{2} \cdot\left(R_{2}-R_{1}\right)}{l^{2} \cdot \cos ^{2} \alpha \cdot \sin ^{2} \alpha}, \\
A=\frac{B^{1 / \operatorname{ctg} \alpha}}{R_{2}} .
\end{gathered}
$$

Substituting equation (19) into equation (16),

$$
\begin{gathered}
\frac{1}{f \cdot \operatorname{ctg} \alpha} \ln \left(\sigma_{L} \cdot f \cdot \operatorname{ctg} \alpha+B\right)=\ln r+\ln \left(\frac{B^{1 / f \cdot \operatorname{ctg} \alpha}}{R_{2}}\right), \\
\sigma_{L}=\frac{\left[\left(r / R_{2}\right)^{f \cdot c t g \alpha}-1\right] \cdot B}{f \cdot \operatorname{ctg} \alpha} .
\end{gathered}
$$

Axial stress $\left(r=R_{1}\right)$ :

$$
\sigma_{L 1}=\frac{\left[\left(R_{1} / R_{2}\right)^{f \cdot \operatorname{ctg} \alpha}-1\right] \cdot B}{f \cdot \operatorname{ctg} \alpha} .
$$

The buffering force:

$$
F_{d}=\sigma_{L 1} \cdot 2 \pi \cdot R_{1} \cdot t=\frac{\left[\left(R_{1} / R_{2}\right)^{f \cdot \operatorname{ctg} \alpha}-1\right] \cdot B \cdot 2 \pi \cdot R_{1} \cdot t}{f \cdot \operatorname{ctg} \alpha} .
$$

It can be seen from equation (24) that the size of the buffer force generated by the thin-walled metal tube is related to the parameters $R_{1}, R_{2}, t, f$, and $\alpha$, which can be changed according to the actual needs to achieve the desired buffer effect.
2.2. Buffering Force of Composite Structure under the HighSpeed Axial Impact. Due to the complexity, inhomogeneity, and uncertainty of aluminum foam, it is difficult to use mathematical models to express the stress changes of aluminum foam during the compression process. The empirical formula is usually gained based on a large number of experiment date for fitting. The failure of the open-cell aluminum foam under the high-speed impact is caused by plastic collapse, whose stress is determined by the yield stress of matrix material. The stress of plastic collapse can be expressed as follows:

$$
\sigma_{p l}=C \cdot \sigma_{l s}\left(\frac{\rho^{*}}{\rho_{s}}\right)^{3 / 2},
$$

where $C$ : constant, measured by test; $\rho^{*}$ : density of aluminum foam; $\rho_{s}$ : density of matrix material; and $\sigma_{l s}$ : yield stress of matrix material.

The buffering force of cylindrical aluminum foam $\left(F_{1}\right)$ when it is in the platform stage under the high-speed impact can be expressed as

$$
F_{1}=A \cdot \sigma_{p l}=C \cdot A \cdot\left(\frac{\rho^{*}}{\rho_{s}}\right)^{3 / 2} .
$$



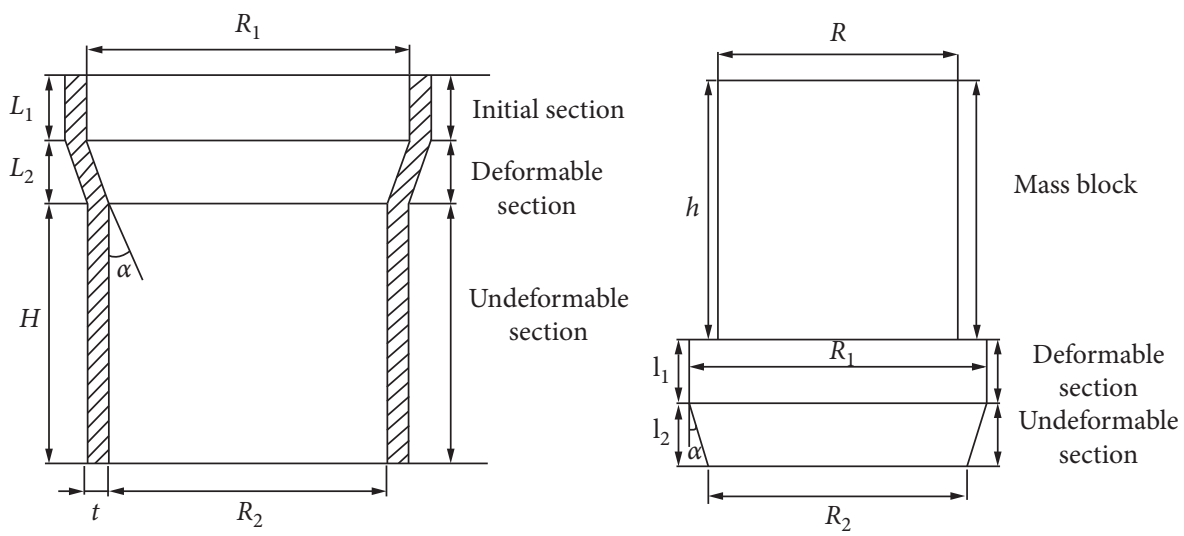

FIGURE 1: Research subject ( $L_{1}$ : length of initial section; $L_{2}$ : length of deformable section; $H$ : length of undeformable section; $t$ : wall thickness of metal tube; $R_{1}$ : inside diameter of initial section; $R_{2}$ : inside diameter of undeformable section; $\alpha$ : half cone angle; $h$ : height of mass block; $R$ : diameter of mass block).

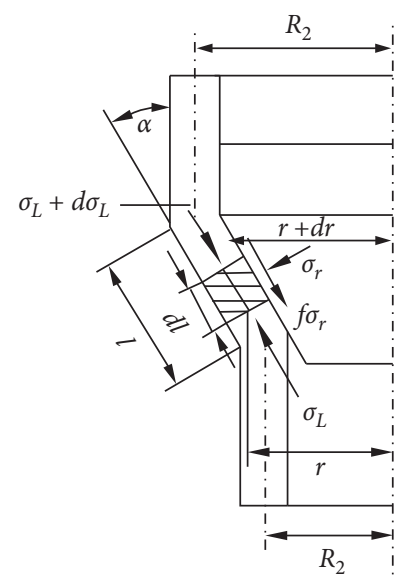

(a)

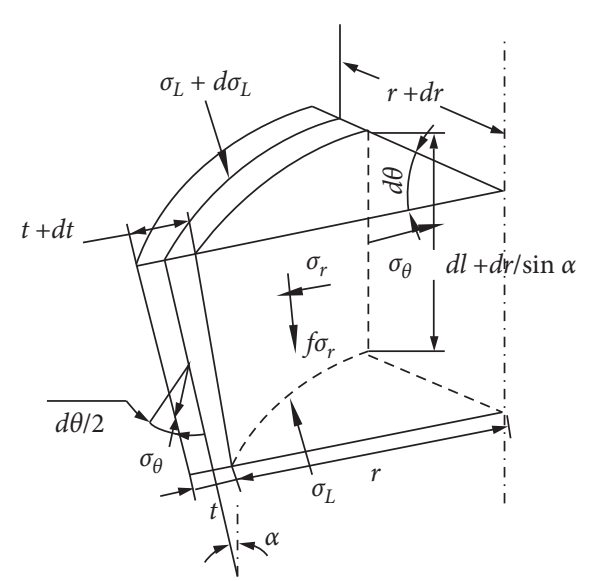

(b)

Figure 2: (a) Stress condition of the metal tube and (b) stress state at arbitrary radius ( $f$ : friction coefficient; $\sigma_{L}$ : axial stress; $\sigma_{\theta}$ : circumferential stress $\sigma_{r}$ : radial stress; $\sigma_{s}$ : yield limit; $\sigma_{d}$ : dynamic yield limit; $\dot{\varepsilon}$ strain rate; $D$, $q$ : constants in Cowper-Symonds equations).

The composite structure of the thin-walled metal tube and aluminum foam could buffer the impact body through radial expansion of the metal tube and plastic deformation of the aluminum foam. It consists of three parts: frustum, thinwalled metal tube, and aluminum foam, with its structure shown in Figure 3. When the mass block impacts the frustum, the metal tube will generate elastic-plastic deformation in the radial direction, forming the axial buffer force. At the same time, the aluminum foam also absorbs part of the energy from impact when compressed, resulting in an axial cushioning force. So, the composite structure can contribute to forming a greater buffering force on the mass block.

Assuming that there is no interaction between the metal tube and the aluminum foam at the axial direction, the buffering force of the composite structure $(F)$ can be expressed as follows:

$$
F=F_{d}+F_{1}
$$

\section{Experiment}

3.1. Structural Parameters of the Projectile. According to the theoretical analysis and the structure of the $125 \mathrm{~mm}$ EPW charge, we designed a thin-walled metal tube buffer filled with aluminum foam that was installed at the front end of the charge and a thin-walled metal tube buffer that was installed at the back end of the charge.

Two buffers were installed into the $125 \mathrm{~mm}$ EPW, as shown in Figures 4 and 5, followed by the fired EPW with artillery. The acceleration date collected by the acceleration sensor 1\# was treated as the overload of the charge during the test, and the acceleration date collected by the acceleration sensor 2\# was treated as the overload of the projectile body during the test. By comparing the acceleration collected by the two sensors, we can verify whether the buffer can effectively reduce the overload of the charge.

The target is made of reinforced concrete. Its length, width, and height are $2.5 \mathrm{~m}, 2.5 \mathrm{~m}$, and $1.5 \mathrm{~m}$, respectively. 


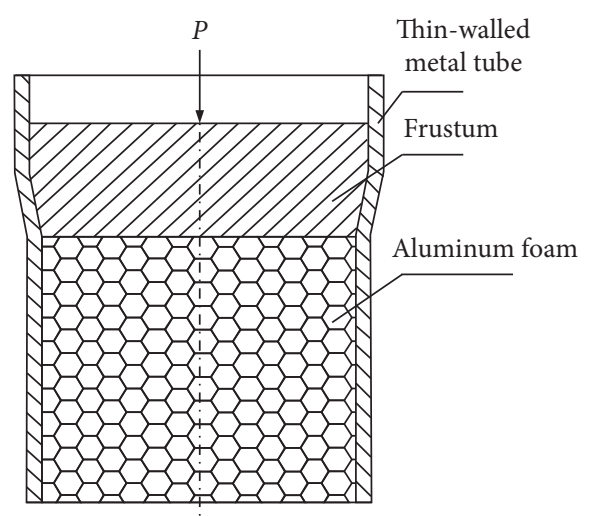

FIgure 3: The composite structure.

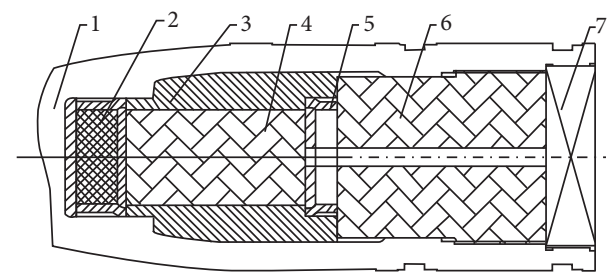

FIgURE 4: The structure of $125 \mathrm{~mm}$ EPW: (1) mian body; (2) buffer No. 1; (3) collar; (4) acceleration sensor 1\#; (5) buffer No. 2; (6) acceleration sensor 2\#; (7) tail.

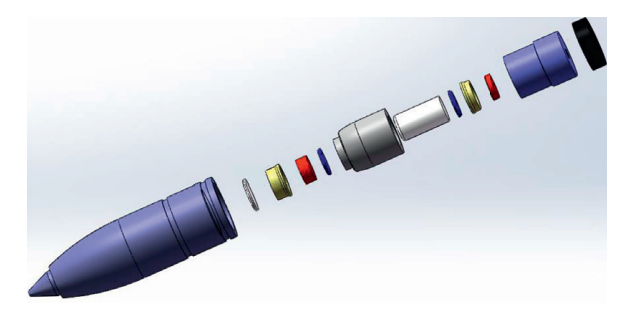

Figure 5: The structure of $125 \mathrm{~mm}$ EPW (three dimensional).

Its compressive strength is $35 \mathrm{MPa}$. The diameter of reinforcement steel in reinforced concrete is $10 \mathrm{~mm}$, and the reinforcement steel ratio was $1 \%$.

All parts were processed and assembled according to the designed drawings with the assembly drawing shown in Figure 6 (the total mass of the projectile was $27.2 \mathrm{~kg}$ ).

Buffer No. 1 consisted of the thin-walled metal tube, aluminum foam, and frustum, as shown in Figures 7 and 8. Its structural parameters are listed in Table 1. Buffer No. 2 consisted of the thin-walled metal tube and frustum, as shown in Figures 9 and 10. Its structural parameters are listed in Table 2.

At the end of the experiment, we designed a device made of concrete to recover the launched projectile so that the data can be collected by the sensors. The installation of the experiment device in the shooting range is shown in Figure 11. The artillery muzzle was 100 meters away from the target. The board was placed behind the target with a thickness of $22 \mathrm{~mm}$ and a total of 11 layers. The recovery device was placed behind and on both sides of the board.

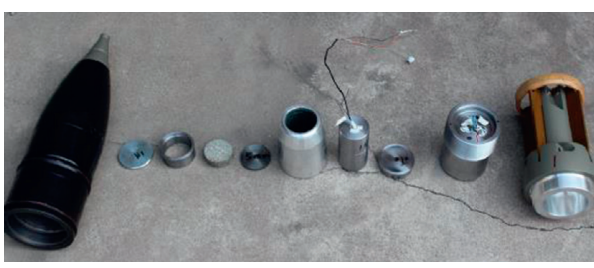

FIgURe 6: Physical drawing of the parts.

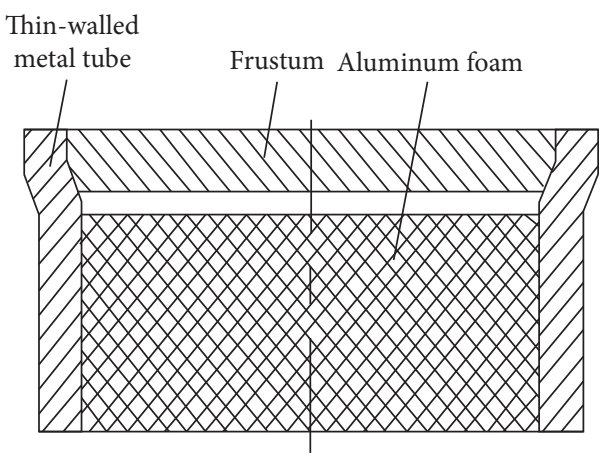

Figure 7: Structure of buffer No. 1.

3.2. Experimental Procedures. The experimental process is as follows:

(1) Measure the quality of each part of the projectile.

(2) Assemble all parts.

(3) Trigger the acceleration sensors and load the projectile into the artillery within five minutes. The artillery is shown in Figure 12.

(4) Fire the projectile.

(5) Measure the hit velocity of the projectile.

(6) Recover the launched projectile and collect the data by sensor $1 \#$ and sensor $2 \#$.

3.3. Experimental Results. The velocity of the projectile was $832 \mathrm{~m} / \mathrm{s}$ when it hit the target. The projectile penetrated into the reinforced concrete target plate, then 11 layers of wood target, and finally into the recovery device with a depth of $0.5 \mathrm{~m}$. The recovered projectile and the penetrated reinforced concrete target are shown in Figure 13.

Two buffers were taken out of the recovered projectile and their structural parameters were measured, as shown in Figure 14. The expanding length of the thin-walled metal tube in buffer No. 1 was $13.785 \mathrm{~mm}$. The thickness of the compressed aluminum foam in buffer No. 1 was $6.215 \mathrm{~mm}$ (before the penetration process: $18 \mathrm{~mm}$ ), the diameter was $56 \mathrm{~mm}$ (before the penetration process: $51.2 \mathrm{~mm}$ ), and the volume compression $62.8 \%$. The expanding length of the thin-walled metal tube in buffer No. 2 was $1.21 \mathrm{~mm}$.

3.3.1. Launching Process. Figures 15(a) and 15(d) show the original and filtered waveforms collected by the acceleration sensor $1 \#$ and acceleration sensor $2 \#$ during the launching 


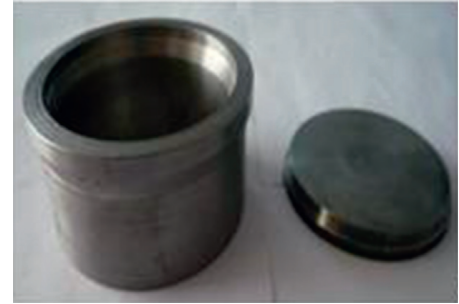

(a)

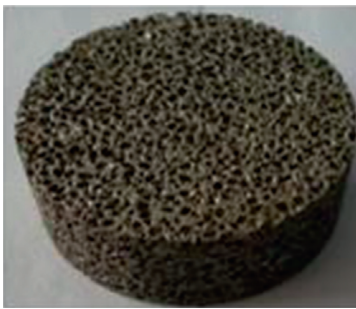

(b)

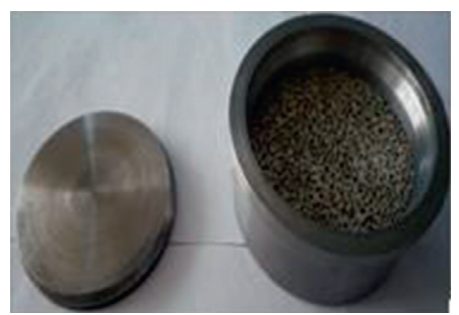

(c)

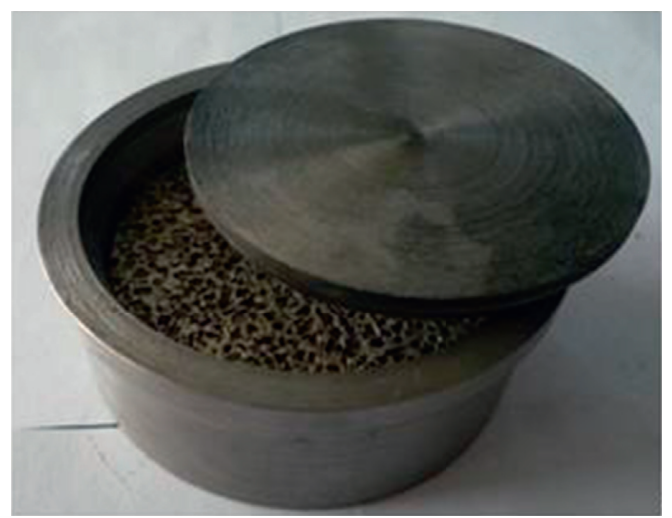

(d)

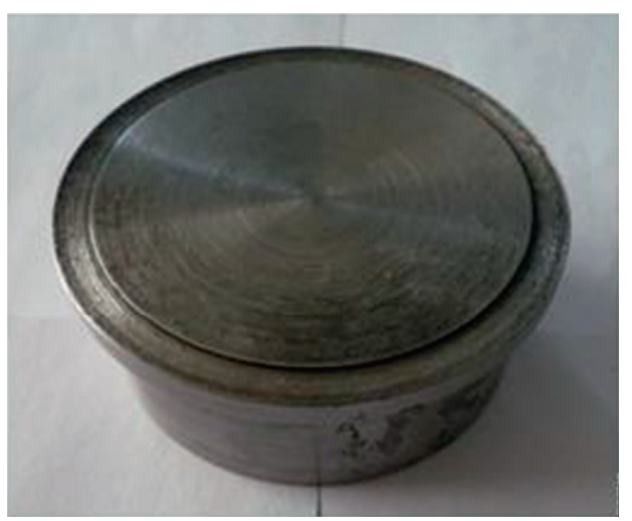

(e)

Figure 8: Physical drawing of buffer No. 1.

Table 1: Structural parameters of buffer No. 1.

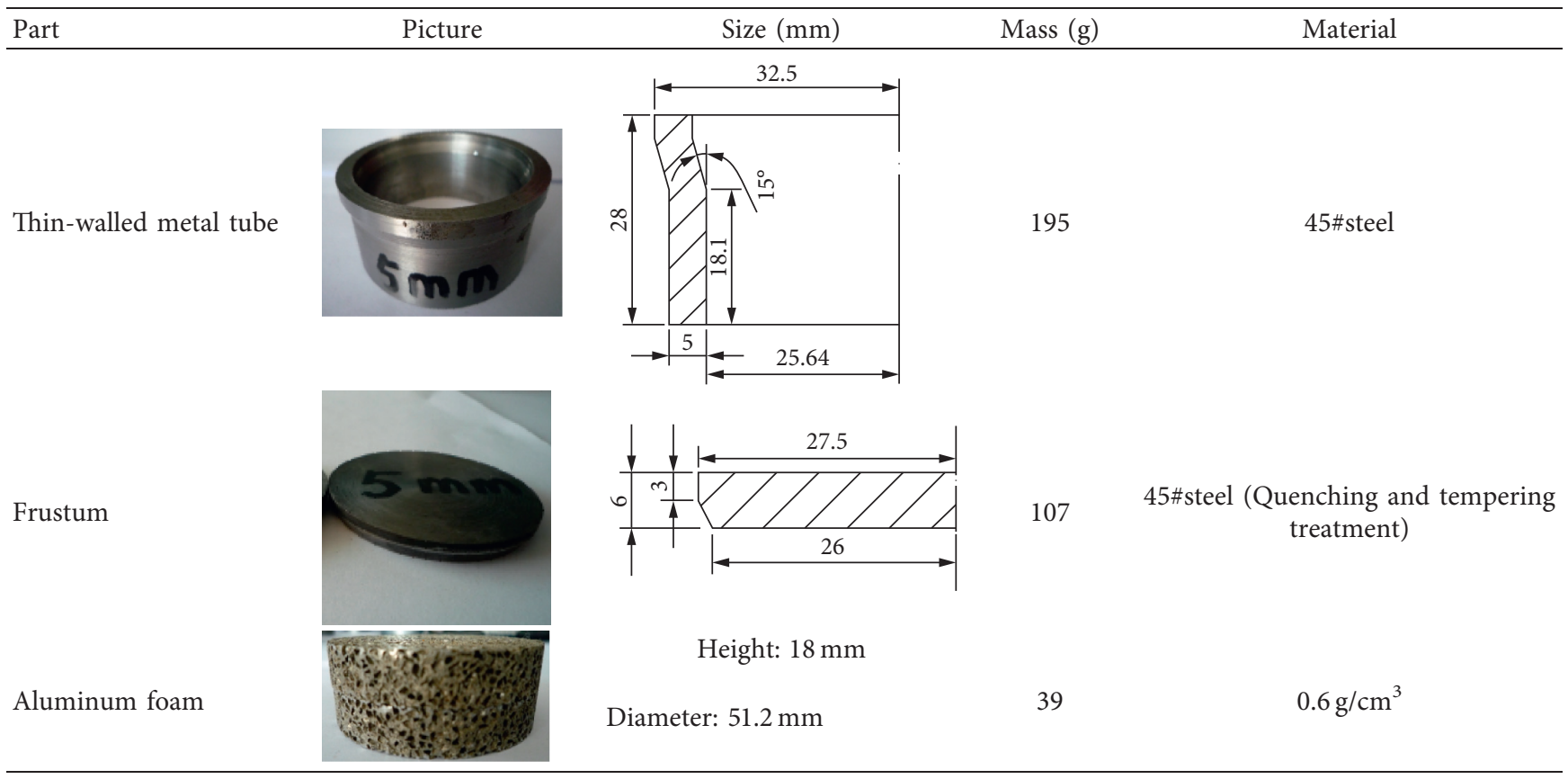

process. From the filtered waveforms, it can be seen that the peak value of the main wave in the waveform chart collected by the sensor $1 \#$ was $11140 \mathrm{~g}$ and the pulse width was $12.45 \mathrm{~ms}$; the peak value of the main wave in the waveform chart collected by the sensor $2 \#$ was $13770 \mathrm{~g}$ and the pulse width was $12.68 \mathrm{~ms}$, which means that the maximum overload received by the charge during the launch process was reduced by $19.1 \%$ after the buffers were installed in the projectile. The trends of the waveforms collected by sensor $1 \#$ and sensor 2\# were basically the same. As the propellant burns, the chamber pressure increased rapidly, reaching a peak in 0.015 seconds, and so did the projectile acceleration value. As the projectile moved forward, the chamber pressure as well as the projectile acceleration value decreased. 


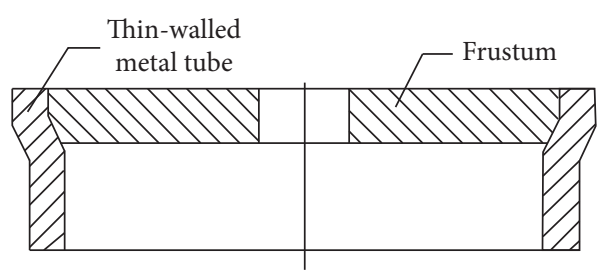

FIgURE 9: Structure of the buffer No. 2.

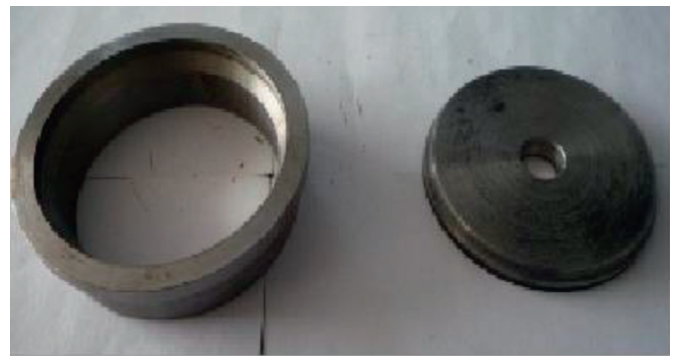

(a)

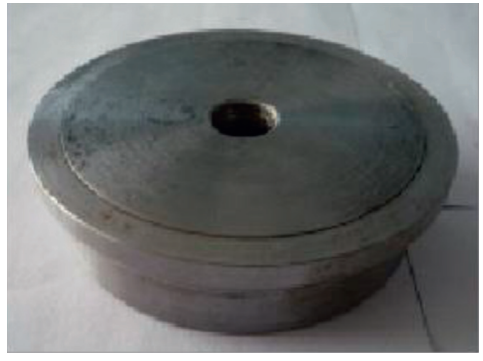

(b)

Figure 10: Physical drawing of buffer No. 2.

TABle 2: Structural parameters of buffer No. 2.

Part
Thin-walled metal
tube
Frustum

When the projectile exited the artillery muzzle, a large amount of gunpowder gas leaked, and the thrust acting at the bottom of the projectile rapidly decreased. Therefore, the acceleration value also quickly dropped to zero. Due to the sudden unloading of the gunpowder gas and the opening of the tail, the combined tensile stress and air resistance led to the negative acceleration value.

3.3.2. Penetration Process. Figures 16(a) and 16(d) show the original and filtered waveforms collected by the acceleration sensor $1 \#$ and acceleration sensor 2\# during the penetration process. From the filtered waveforms, it can be seen that the peak value of the main wave in the waveform chart collected by the sensor 1 \# was $40380 \mathrm{~g}$ and the pulse width was $4.62 \mathrm{~ms}$; the peak value of the main wave in the waveform chart collected by the sensor 2\# was $69010 \mathrm{~g}$ and the pulse width was $0.77 \mathrm{~ms}$, which showed that the maximum overload received by the charge during the penetration process was reduced by $41.5 \%$ after the buffers were installed in the projectile.

It could be seen from Figure 16 that the overload of the sensor $1 \#$ during the penetration process changed more smoothly, while the overload of the sensor 2\# during the 


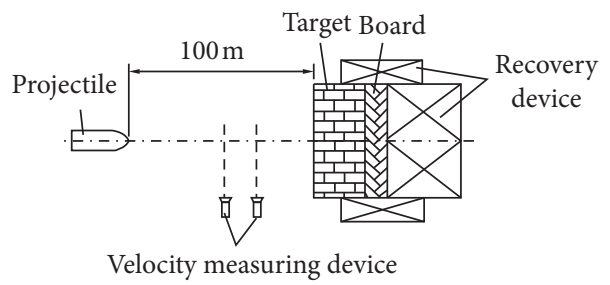

FIgURE 11: Experiment device installation.

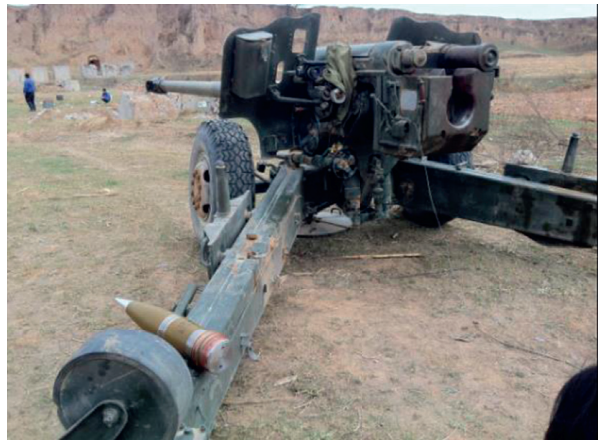

Figure 12: The artillery.

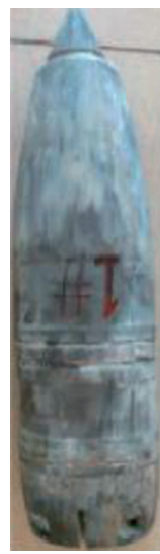

(a)

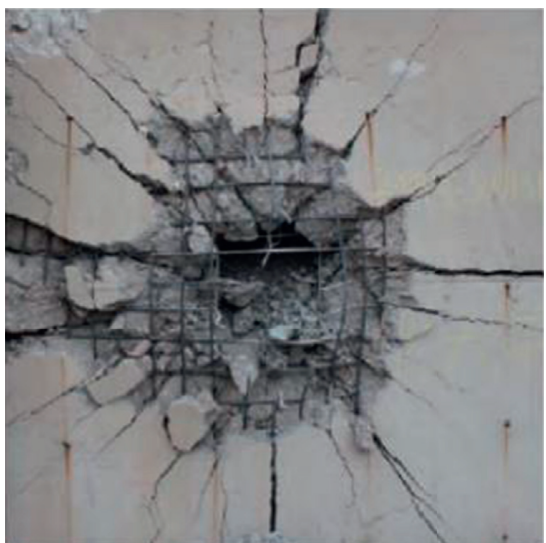

(b)

Figure 13: (a) The projectile; (b) the reinforced concrete target.

penetration process changed more drastically. A comparison of Figures 15 and 16 can show that the projectile and its charge were subjected to a larger overload during the penetration process than during the launching process.

Based on the test results, we could conclude that (1) at the end of the experiment, buffer No. 1 produced a large amount of plastic deformation, while buffer No. 2 produced only a small amount of plastic deformation, which indicates that buffer 1 plays the major role in buffering and energy absorption; (2) after two buffers were installed at both ends of the charge, the maximum overload that the charge was subjected to during the launch and penetration process was significantly reduced, and the change in overload was also more gentle.
The above test results show that the buffer with the composite structure can effectively reduce the impact load of the $125 \mathrm{~mm}$ EPW charge during the penetration process. Through reasonable structural design, the composite structure of foamed aluminum-filled thin-walled metal tube can be applied to the protection of high-speed projectile charges.

In the published research results, there is no research on applying this composite structure of aluminum foam-filled metal thin-walled tube for the protection of projectile charges. At present, the relevant research mainly covers the following two areas: one is the study on the low- and medium-speed impact represented by the drop-weight impact method; the second is the High-speed impact research 


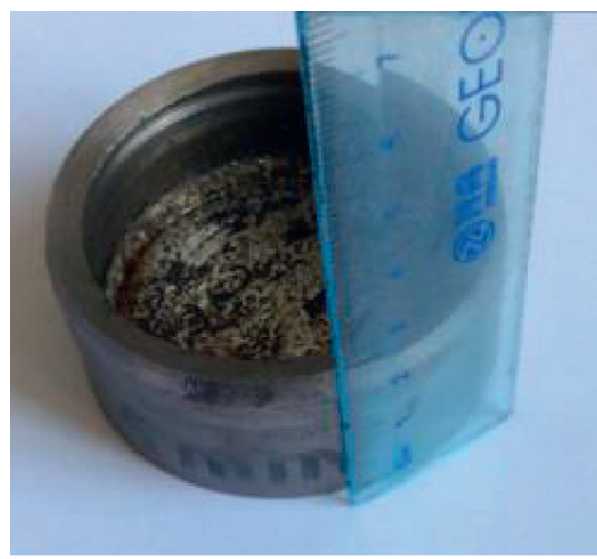

(a)

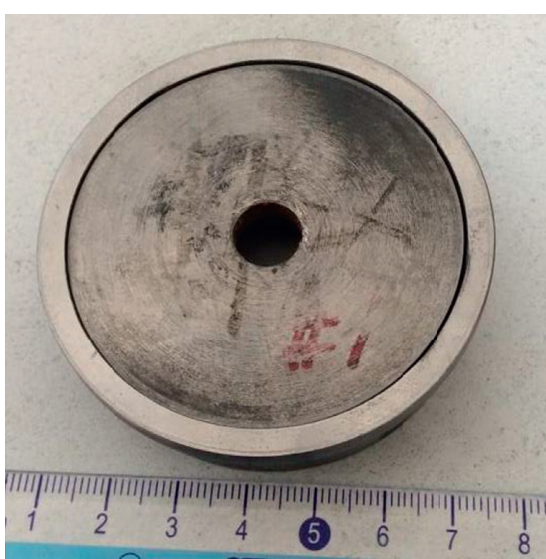

(b)

FiguRE 14: Buffers after the penetration: (a) buffer No. 1; (b) buffer No. 2.

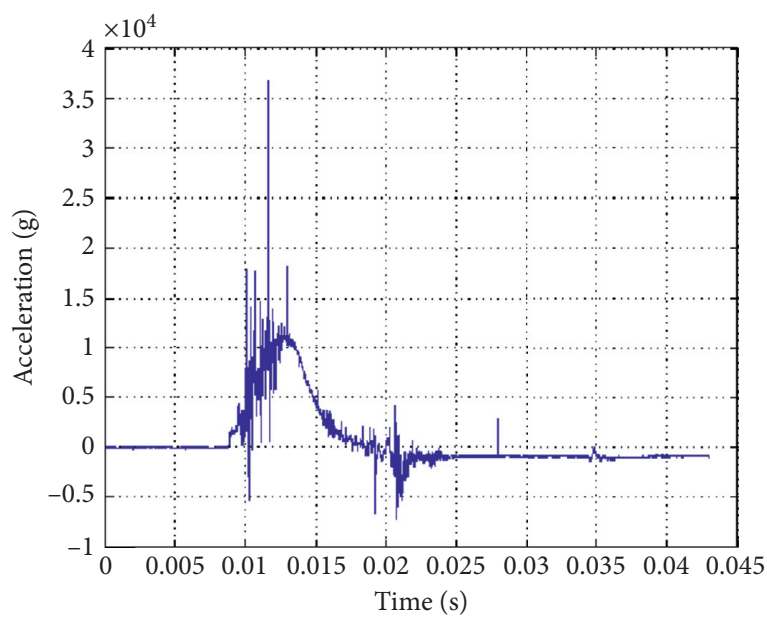

(a)

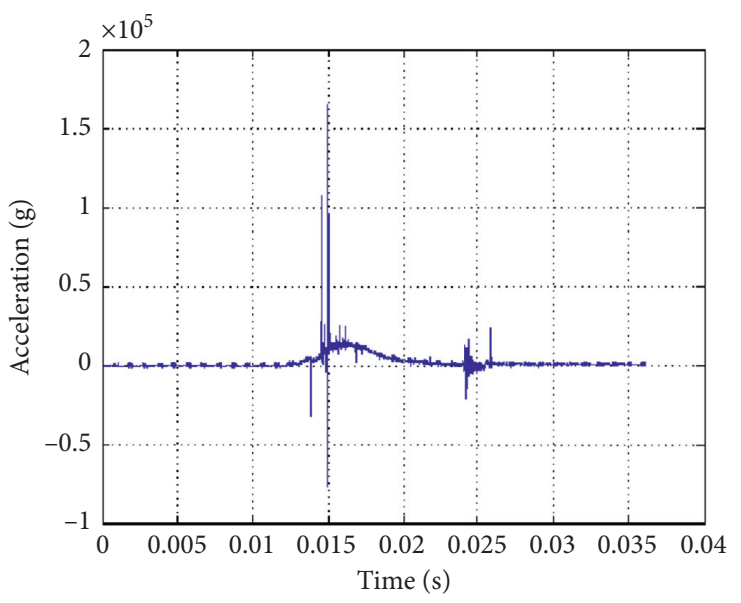

(c)

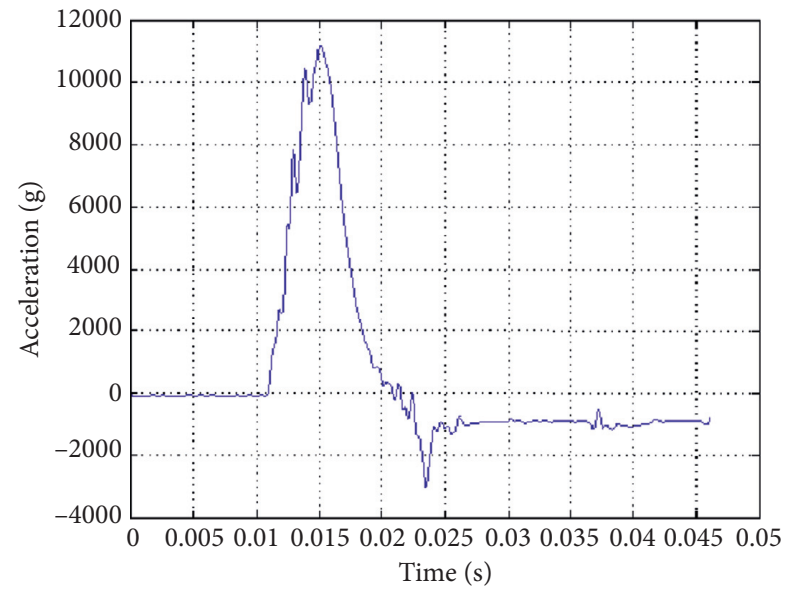

(b)

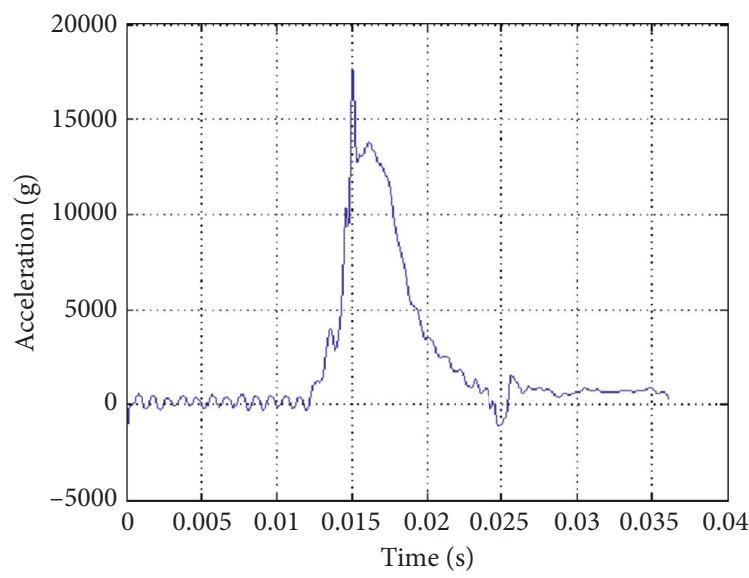

(d)

FIGURE 15: Waveform chart in launching process: (a) the original signal collected by acceleration sensor 1\#; (b) the filtered signal collected by acceleration sensor 1\#; (c) the original signal collected by acceleration sensor 2\#; (d) the filtered signal collected by acceleration sensor 2\#.

represented by Hopkinson bar technology (SHPB). The impact velocity of the composite structure in these two test methods did not reach the penetration velocity of the projectile (above $800 \mathrm{~m} / \mathrm{s}$ ). For the protection of high-speed projectile charges, a buffer device made of nylon or rubber are often installed at the front end of the charge. 


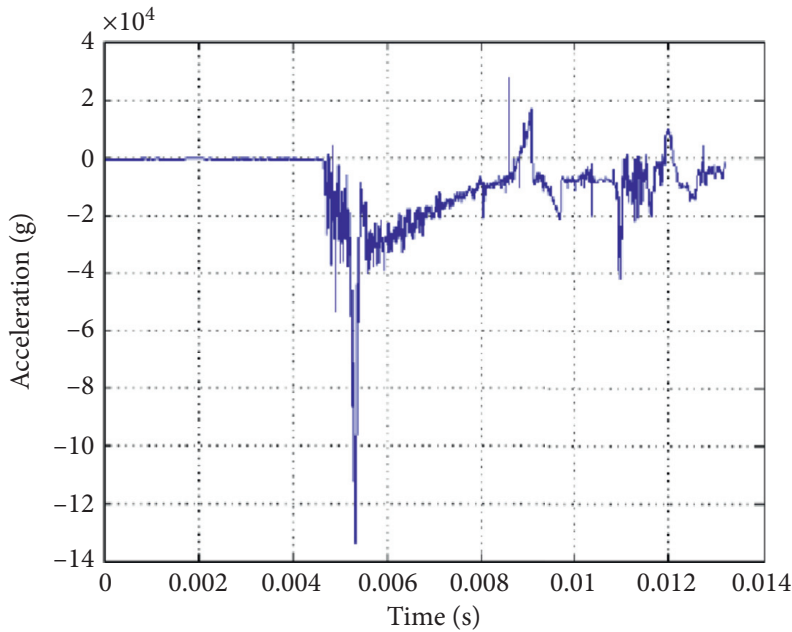

(a)

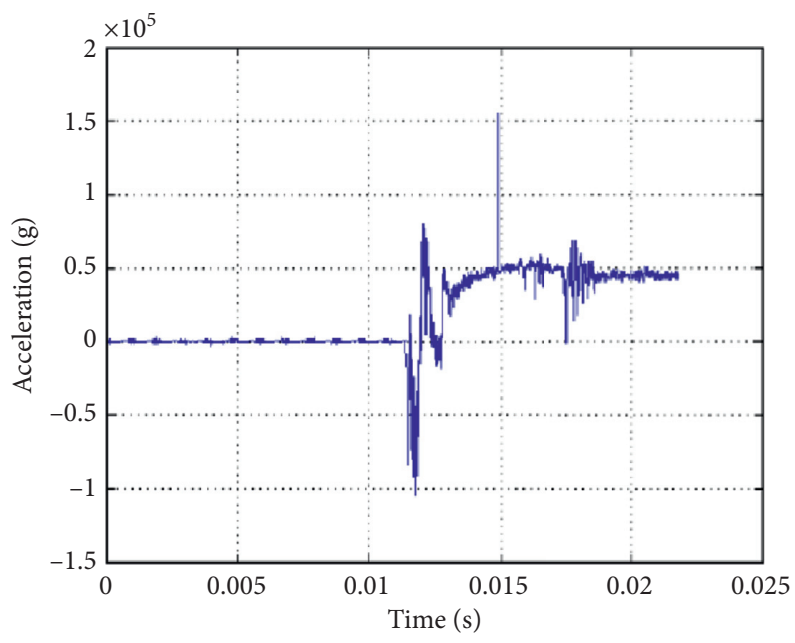

(c)

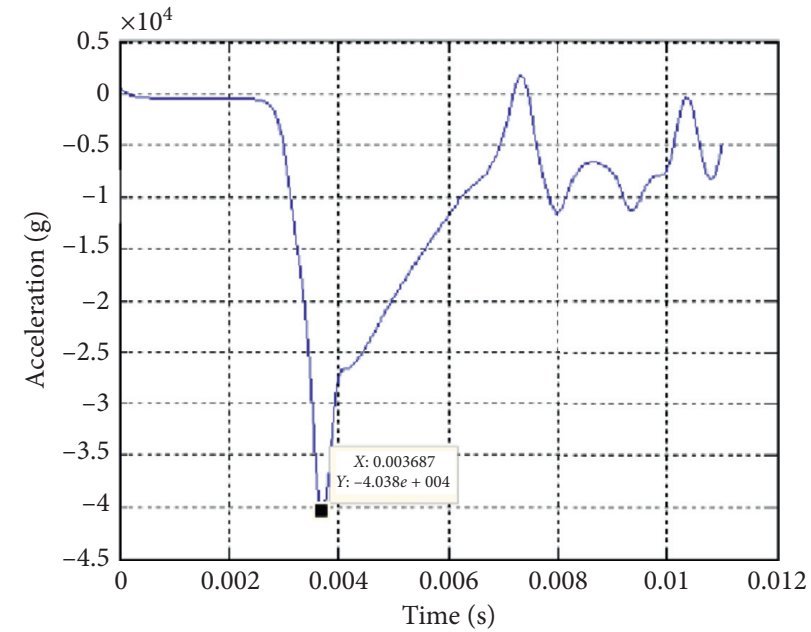

(b)

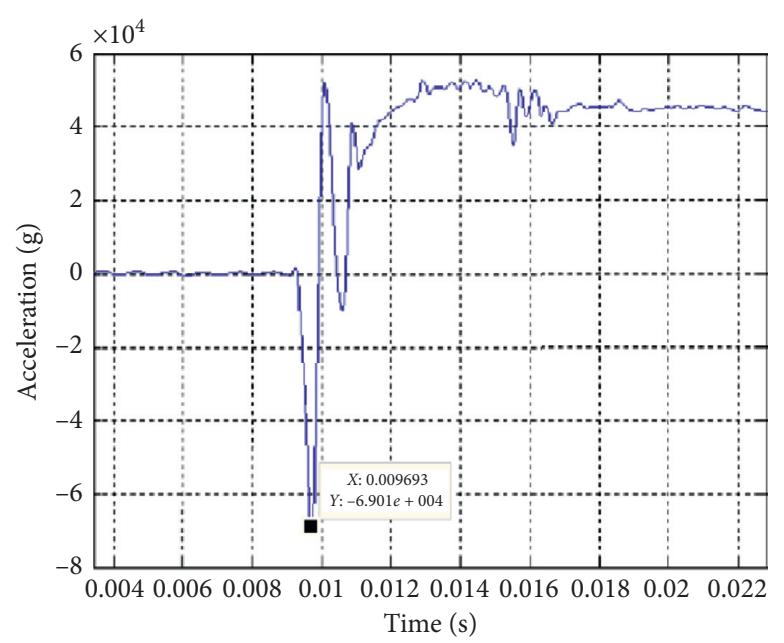

(d)

FIGURE 16: Waveform chart in the penetration process: (a) the original signal collected by acceleration sensor 1\#; (b) the filtered signal collected by acceleration sensor $1 \#$; (c) the original signal collected by acceleration sensor 2\#; (d) the filtered signal collected by acceleration sensor 2\#.

\section{Conclusion}

In terms of theoretical and practical aspects, the main conclusions of this work can be summarized as follows:

(1) A theoretical analysis was made on the dynamic response of the thin-walled metal tube and composite structure of aluminum foam-filled thin-walled metal tube under the impact of high-speed mass block was theoretically analyzed, together with some assumptions put forward. Based on these assumptions, we derived the expression of the buffer force of the thin-walled metal tube $\left(F_{d}\right)$ and the composite structure aluminum foam and thin-walled metal tube $(F)$ through the formula derivation.

(2) According to the results of theoretical analysis and combining the structure of the $125 \mathrm{~mm}$ EPW, a design was conducted on a buffer with a composite structure of foamed aluminum-filled thin-walled metal tubes with specific dimensions. It was then installed at the front end of the charge. The penetrating concrete test was carried out with the projectile equipped with the buffer. The test results show that the overload on the charge was significantly reduced after the buffer was installed. It is obvious that this composite structure can be applied to the protection of high-speed penetration projectile charges.

\section{Data Availability}

The data used to support the findings of this study are included within the article.

\section{Conflicts of Interest}

The authors declared that they have no conflicts of interest regarding the publication of this work. 


\section{Acknowledgments}

This work was financially supported by the Nature Science Foundation of Shanxi Province China (no. 201801D121107).

\section{References}

[1] H. Muthig and W. Arnold, "Sensitivity and Structural Investigations On quasi-Static and shock loaded. KS22a high explosive," in Proceedings of the Insensitive Munitions \& Energetic Materials Technology Symposium, San Francisco, CA, USA, November 2004.

[2] A. A. A. Alghamdi, "Collapsible impact energy absorbers: an overview," Thin-Walled Structures, vol. 39, no. 2, pp. 189-213, 2001.

[3] W. Abramowicz, "Thin-walled structures as impact energy absorbers," Thin-Walled Structures, vol. 41, no. 1, pp. 91-107, 2003.

[4] S. Salehghaffari, M. Panahipoor, and M. Tajdari, "Controlling the axial crushing of circular metal tubes using an expanding rigid ring press fitted on top of the structure," International Journal of Crashworthiness, vol. 15, no. 3, pp. 251-264, 2010.

[5] S. P. Santosa, T. Wierzbicki, A. G. Hanssen, and M. Langseth, "Experimental and numerical studies of foam-filled sections," International Journal of Impact Engineering, vol. 24, no. 5, pp. 509-534, 2000.

[6] M. Shakeri and S. Salehghaffari, "Expansion of circular tubes by rigid tubes as impact energy absorbers: experimental and theoretical investigation," International Journal of Crashworthiness, vol. 5, no. 3, pp. 493-501, 2007.

[7] K. Ramajeyathilagam, C. P. Vendhan, and V. Bhujanga Rao, "Non-linear transient dynamic response of rectangular plates under shock loading," International Journal of Impact Engineering, vol. 24, no. 10, pp. 999-1015, 2000.

[8] G. H. Majzoobi, R. Azizi, and A. Alavi Nia, "A three-dimensional simulation of shot peening process using multiple shot impacts," Journal of Materials Processing Technology, vol. 164165, pp. 1226-1234, 2005. 